\title{
JUNO: A Multipurpose Underground Precision Neutrino Detector
}

\section{Qingmin Zhang ${ }^{\mathbf{1}}$}

Department of Nuclear Science and Technology, School of Energy and Power Engineering, Xi'an Jiaotong University, Xi'an 710049, China

E-mail: zhangqingminemail.xjtu. edu. cn

On behalf of JUNO collaboration

The Jiangmen Underground Neutrino Observatory (JUNO) is under construction in China to mainly determine the neutrino mass hierarchy $(\mathrm{MH})$ by detecting reactor anti-neutrinos. JUNO consists of central detector, veto detector and calibration system. Its central detector is a $20 \mathrm{kton}$ liquid scintillator (LS) detector and its calibration system is designed to measure the energy of neutrinos with an unprecedented energy resolution of 3\%@1MeV and an energy nonlinearity better than $1 \%$. Veto detector, consisting of water Cherenkov detector and top tracker, is used to maximally remove the background events due to cosmic-rays. Due to its large scale and best performance, JUNO will be an exceptional multipurpose detector with rich physical potentials, such as precise measurements of neutrino oscillation parameters, geo-neutrinos, astrophysical neutrinos, sterile neutrinos, dark matter searches, proton decay and other exotic searches.

The 39th International Conference on High Energy Physics (ICHEP2018)

4-11 July, 2018

Seoul, Korea

\footnotetext{
${ }^{1}$ Speaker

(C) Copyright owned by the author(s) under the terms of the Creative Commons

Attribution-NonCommercial-NoDerivatives 4.0 International License (CC BY-NC-ND 4.0).
} 


\section{JUNO Introduction}

Non-zero and large $\theta_{13}$ discovery ${ }^{[1]}$ opens a door to neutrino Mass Hierarchy (MH), the Jiangmen Underground Neutrino Observatory (JUNO) was proposed in 2008 and approved in 2013. Its site location is optimized to have the best sensitivity for the $\mathrm{MH}$ determination, which is at $53 \mathrm{~km}$ from both the Yangjiang and Taishan Nuclear Power Plants ${ }^{[2]}$. As a multiple-purpose neutrino observatory, JUNO also has other rich physical potentials ${ }^{[3]}$. JUNO Collaboration was established in July 2014, and there were 72 institutions and 593 collaborators in JUNO collaboration by July, 2018. JUNO civil construction started in 2014 and data-taking is expected to be in the year of 2021.

\section{JUNO Detector}

JUNO is an underground Liquid Scintillator (LS) detector with an overburden of 700-meter rock for shielding of cosmic rays. As a multiple-purpose neutrino observatory, JUNO consists of Central Detector (CD), Veto Detector and Calibration System, as shown in Fig.1. Veto detector consists of Water Cherenkov detector and top tracker. ${ }^{[2]}$

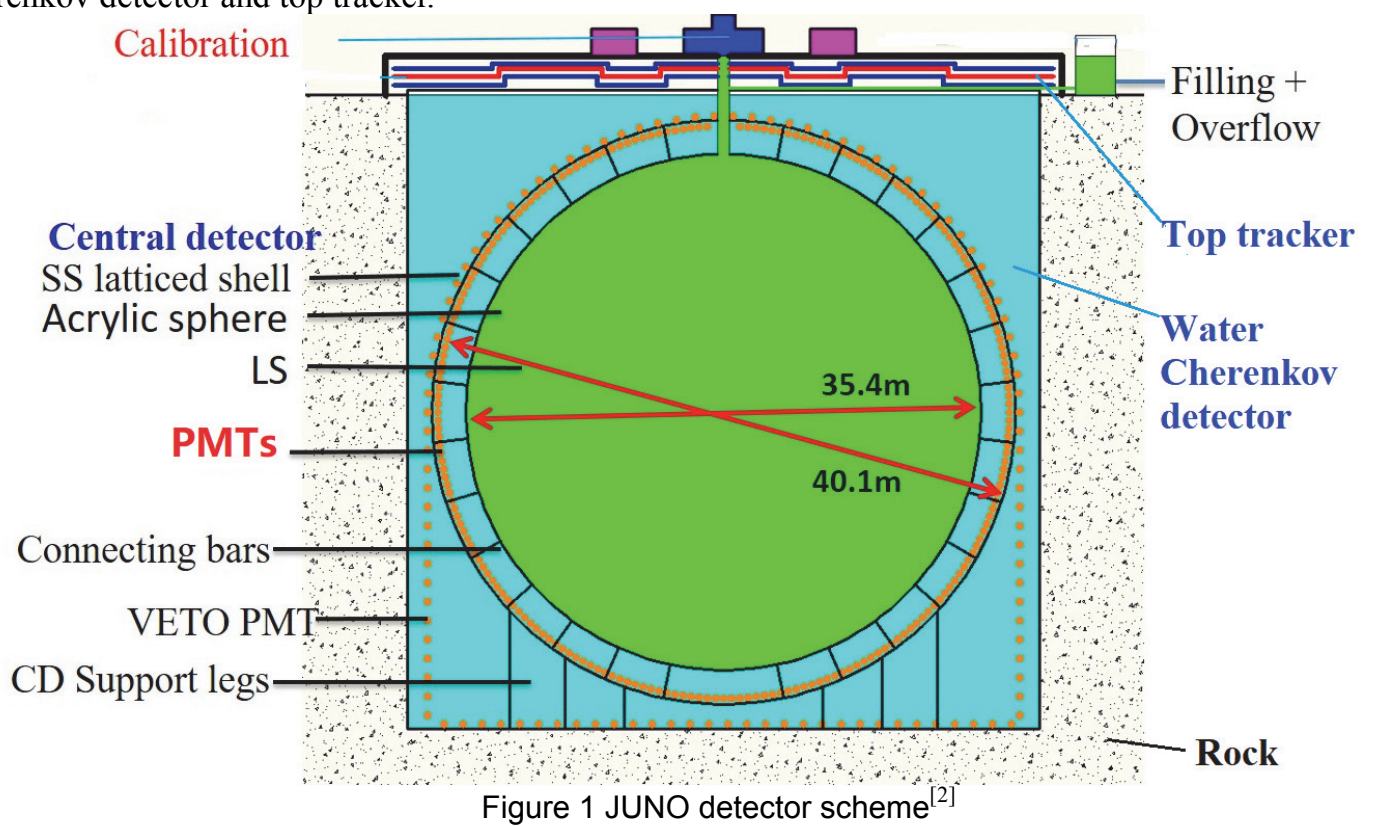

\subsection{Central Detector}

JUNO central detector (CD), an acrylic sphere with a diameter of $35.4 \mathrm{~m}$, is filled with liquid scintillator(LS) and equipped with about 18000 high QE 20-inch PMTs and 25000 3-inch PMTs to measure the energy of neutrinos. It' worth metionding that 3-inch PMTs will be used for better timing and higher saturation energy. An acrylic spherical shell with a diameter of $35.4 \mathrm{~m}$ and a stainless steellatticed shell with a diameter of $40.1 \mathrm{~m}$ will be constructed for LS container and PMT deployment respectively. JUNO CD will be the largest Liquid Scintillator detector in the world to achieve an unprecedented energy resolution of 3\%@1 MeV. Liquid Scintillator and 20-inch PMTs are very critical for JUNO CD, and they will be introduced below. ${ }^{[4]}$

Liquid Scintillator serves as the target material for reactor anti-neutrino detection. Its recipe consists of Linear Alkyl Benzene (LAB) as solvent, $2.5 \mathrm{~g} / \mathrm{L}$ 2,5-diphenyloxazole (PPO) as the fluor and 1-4mg/L p-bis-(o-methylstyryl)-benzene (bis-MSB) as the wavelength shifter ${ }^{[5]}$. For the unprecedented energy resolution, light yield is required to be greater than 10000 photons/ $\mathrm{MeV}$ and attenuation length should be longer than $20 \mathrm{~m}$ at $430 \mathrm{~nm}$ wavelength. Furthermore, the radioactive concentrations of ${ }^{238} \mathrm{U},{ }^{232} \mathrm{Th}$ and ${ }^{40} \mathrm{~K}$ should be below $10^{-15} \mathrm{~g} / \mathrm{g}, 10^{-15} \mathrm{~g} / \mathrm{g}$ and $10^{-17} \mathrm{~g} / \mathrm{g}$ respectively. Accordingly, LS will be purified by distillation, water extraction and steam stripping ${ }^{[4]}$.

PMTs will be used to detect the scintillation photons from IBD events in LS. Additionally, about 2400 20-inch PMTs are also used to detector Cherenkov light in water Cherenkov detector, so 2000020 inch PMTs are needed in total. 15000 MCP-PMTs are supplied by North Night Vision Technology (NNVT) and 5,000 R12860 PMTs are from Hamamatsu. Generally, the PMTs from the two companies are 
similar in performances, and it's worth mentioning the PMT's detection efficiency (= quantum efficiency $\times$ collection efficiency) is required to be $27 \%$ on average and $24 \%$ on minimum. However, NNVT PMTs have a lower after-pulse percentage and glass radioactivity for lower background, while Hamamatsu PMTs have a shorter transit-time spread for better vertex reconstruction due to its better timing. Protection cover is designed to prevent chain implosion reaction for all 20-inch PMTs. ${ }^{[4]}$

\subsection{Veto System}

The cosmic ray muon induced backgrounds are the main backgrounds and hard to remove. In order to reduce the experimental backgrounds, it's not enough that the neutrino detector is placed in deep underground, so a veto system is used to tag muons. Cosmogenic isotopes reduction $\left({ }^{9} \mathrm{Li} /{ }^{8} \mathrm{He}\right)$ requires precise muon track. The remaining energetic cosmic ray muons can still produce a large number of neutrons in the rocks and other detector materials surrounding the central detector. These neutrons can produce fast neutron background in the central detector which mimics the inverse beta decay signal. The muons should be detected with high efficiency and precise tracking performance for the purpose of background reduction. Additionally, at least 2 meters of water surrounding the central detector is needed to shield the neutrons and the natural radioactivity from the surrounding rock. ${ }^{[2]}$

Accordinlgy, the veto detector is designed to consist of water Cherenkov detector and top tracker. Water Cherenkov detector is made of 20-30 kton ultrapure water with radioactivity below $0.2 \mathrm{~Bq} / \mathrm{m}^{3}$, which is supplied and maintained by a circulation system. The Cherenkov light is detected by $\sim 240020-$ inch PMTs and its detection efficiency is greater than 95\%. Top tracker is made of reused plastic scintillators from OPERA experiment and it covers half of water pool on the top with 3-layers configuration. Each module is readout at both ends by multi-anode photomultipliers. ${ }^{[2]}$

\subsection{Calibration System}

Due to its structure and big volume, JUNO energy response strongly depends on the event's position, so a complete calibration complex has been designed for full volume coverage ${ }^{[8]}$. Automatic Calibration Unit (ACU), Cable Loop System (CLS) and Guide Tube Control System (GTCS) will be combined to deliver ${ }^{40} \mathrm{~K}$ source to specified positions in one plane for non-uniformity correction, Remotely Operated under-liquid-scintillator Vehicles (ROV) will be used for full volume scan when needed. On the other hand, ACU with multiple sources at $\mathrm{CD}$ center will be used for nonlinearity correction. The radiation sources include neutron sources $\left({ }^{241} \mathrm{Am}-\mathrm{Be},{ }^{241} \mathrm{Am}-{ }^{13} \mathrm{C},{ }^{241} \mathrm{Pu}-{ }^{13} \mathrm{C},{ }^{252} \mathrm{Cf}\right.$ ), positron sources $\left({ }^{22} \mathrm{Na},{ }^{68} \mathrm{Ge},{ }^{40} \mathrm{~K}\right.$, ${ }^{90} \mathrm{Sr}$ ) and gamma sources $\left({ }^{40} \mathrm{~K},{ }^{54} \mathrm{Mn},{ }^{60} \mathrm{Co},{ }^{137} \mathrm{Cs}\right)$ will be used for calibration. Finally, the energy can be measured accurately. Simulation shows the energy bias is less than $0.1 \%$ and the energy resolution is better than $3.0 \%$ for uniformly distributed mono-energy $\mathrm{e}^{+}$events ${ }^{[4,5]}$.

\section{JUNO Physics Goals and Potentials}

Due to its large scale and best performance, JUNO will be an exceptional detector and it has rich physics potentials.

\subsection{Mass Hierarchy}

JUNO is designed to determine the neutrino MH using precision spectral measurements of reactor antineutrino oscillations, because reactor neutrino oscillation probability is independent of CP phase and $\theta_{23}$. The electron antineutrino survival probability in vacuum can be written as:

$$
\begin{aligned}
P_{\bar{v}_{e} \rightarrow \bar{v}_{e}}= & 1-\cos ^{4} \theta_{13} \sin ^{2} 2 \theta_{12} \sin ^{2}\left(\Delta_{21}\right) \\
& -\sin ^{2} 2 \theta_{13} \sin ^{2}\left(\left|\Delta_{31}\right|\right) \\
& -\sin ^{2} \theta_{12} \sin ^{2} 2 \theta_{13} \sin ^{2}\left(\Delta_{21}\right) \cos \left(2\left|\Delta_{31}\right|\right) \\
& \pm \frac{\sin ^{2} \theta_{12}}{2} \sin ^{2} 2 \theta_{13} \sin \left(2 \Delta_{21}\right) \sin \left(2\left|\Delta_{31}\right|\right)
\end{aligned}
$$

where $\Delta_{i j}=\Delta m_{i j}^{2} L / 4 E$, in which $L$ is the baseline, $E$ is the antineutrino energy and the \pm sign in the last term of Eq. (1) is decided by the MH with plus sign for the normal MH and minus sign for the inverted $\mathrm{MH}^{[6]}$.

The determination of the neutrino MH is of great importance in neutrino physics, since the MH pro- 
vides a crucial input for future searches of neutrinoless double beta decays, observation of supernova neutrino bursts, cosmological probe of neutrino properties, and model building of the neutrino masses and flavor mixing. Thanks to the relatively large $\theta_{13}$ discovered in recent reactor and accelerator neutrino experiments, precise measurements of the reactor antineutrino spectrum at a medium baseline of about 50 $\mathrm{km}$ can probe the interference effect of two fast oscillation modes (i.e., oscillations induced by $\Delta \mathrm{m}_{21}^{2}$ and $\Delta \mathrm{m}^{2}{ }_{13}$ ) and sensitive to the neutrino MH. JUNO will observe 60 Inverted Beta Decay events per day with about $6 \%$ backgrounds. The sensitivity of $\sim 3 \sigma$ can be achieved during 6 years of running ${ }^{[3]}$.

\subsection{Measurement of Oscillation Parameters}

Precision measurement of the oscillation parameters and test of the standard three-neutrino framework constitute another important goal of the JUNO experiment. Due to good energy resolution and proper baseline, JUNO will also allow a detailed study of various aspects of neutrino oscillations, including the extractions of $\Delta \mathrm{m}_{21}^{2}, \Delta \mathrm{m}_{\text {ee }}^{2}$ and $\sin ^{2} \theta_{12}$. As shown in Table 1, JUNO can measure the three oscillation parameters $\sin ^{2} \theta_{12}, \Delta \mathrm{m}_{21}^{2}$ and $\Delta \mathrm{m}_{\text {ee }}^{2}$ to world-leading levels of $0.7 \%, 0.6 \%$ and $0.5 \%$ respectively ${ }^{[3]}$.

Table 1 Precision of $\sin ^{2} \theta_{12}, \Delta \mathrm{m}_{21}^{2}$ and $\Delta \mathrm{m}^{2}$ ee from the nominal setup to those including additional systematic uncertainties. The systematics are added one by one from left to right ${ }^{[3]}$.

\begin{tabular}{|c|c|c|c|c|c|}
\hline & Nominal & $+\mathrm{B} 2 \mathrm{~B}(1 \%)$ & $+\mathrm{BG}$ & $+\operatorname{EL}(1 \%)$ & $+\mathrm{NL}(1 \%)$ \\
\hline $\sin ^{2} \theta_{12}$ & $0.54 \%$ & $0.60 \%$ & $0.62 \%$ & $0.64 \%$ & $0.67 \%$ \\
\hline$\Delta \mathrm{m}_{21}^{2}$ & $0.24 \%$ & $0.27 \%$ & $0.29 \%$ & $0.44 \%$ & $0.59 \%$ \\
\hline$\left|\Delta \mathbf{m}^{2}{ }_{\text {ee }}\right|$ & $0.27 \%$ & $0.31 \%$ & $0.31 \%$ & $0.35 \%$ & $0.44 \%$ \\
\hline
\end{tabular}

Utilizing these above measurements, JUNO can also help test the unitarity relation and the mass sum rule to the levels of around $1.2 \%$ and $1.8 \%$, respectively. Moreover, JUNO would be the first experiment to simultaneously observe neutrino oscillations from two different frequencies, and be the first experiment to observe more than two oscillation cycles ${ }^{[3]}$.

\subsection{Neutrino Astrophysics and Others}

Neutrinos from the earth escape freely and bring the information about $\mathrm{U}$, Th and $\mathrm{K}$ abundances and their distributions. Due to its largest LS size, the expected geo-neutrino rate in JUNO is $\sim 1.1 /$ day. Within the $1^{\text {st }}$ year, JUNO will record more geo-neutrino events than all other detectors and JUNO will be the most precise experiment for geo-neutrino study ${ }^{[3]}$. In the meanwhile, JUNO is also attractive for other neutrino astrophysics, such as supernova neutrinos, diffuse supernova neutrinos, solar neutrinos and atmospheric neutrinos ${ }^{[3]}$.

Beside these, additional physics is also rich in JUNO, such as sterile neutrinos, dark matter searches, proton decay and other exotic searches ${ }^{[3]}$.

\section{Summary}

JUNO is a multipurpose underground precision neutrino detector, which is a very active R\&D program and will achieve design goals. JUNO will determine mass hierarchy (3-4 $\sigma$ in 2027) and 3 oscillation parameters to $<1 \%$ level, with other rich physics potentials, such as supernova, geo-neutrino, solar neutrino, sterile neutrino.JUNO detector R\&D and fabrication are progressing smoothly, aiming at data-taking in 2021.

\section{References}

[1] F. P. An et al., Observation of Electron-Antineutrino Disappearance at Daya Bay [J], Phys. Rev. Lett. 108, 171803.

[2] T. Adam et al., JUNO Conceptual Design Report [R], https://arxiv.org/abs/1508.07166.

[3] F.P. An et al., Neutrino physics with JUNO [J], J. Phys. G: Nucl. Part. Phys. 43 (2016) 030401 (188pp).

[4] Q.M. Zhang et al., JUNO Central Detector and its Calibration System [J],38th International Conference on High Energy Physics, Chicago, USA,3-10 August,2016, Proceedings of Science, PoS(ICHEP2016)967.

[5] F.Y. Zhang, Calibration for energy non-linearity and resolution [R], Beijing: IHEP, 2018.

[6] L. Zhan, Y.F. Wang, Determination of the neutrino mass hierarchy at an intermediate baseline [J], Phys. Rev. D 78, 111103 (2008). 